\title{
DETECÇÃO DE FUNGOS ENDOFÍTICOS EM SEMENTES DE CAUPI PROVENIENTES DE SERRA TALHADA E DE CARUARU, ESTADO DE PERNAMBUCO
}

\author{
ANTONIA A. C. RODRIGUES \& MARIA MENEZES \\ Departamento de Agronomia-Fitossanidade, Universidade Federal Rural de Pernambuco, \\ CEP 52171-900, Recife, PE
}

(Aceito para publicação em 02/07/2002)

Autor para correspondência: Antonia Alice Costa Rodrigues

RODRIGUES, A.A.C. \& MENEZES, M. Detecção de fungos endofíticos em sementes de caupi provenientes de Serra Talhada e de Caruaru, Estado de Pernambuco. Fitopatologia Brasileira 27:532-537. 2002.

\section{RESUMO}

O presente trabalho teve como objetivo detectar e identificar fungos endofíticos em sementes de caupi (Vigna unguiculata), IPA-201, IPA-202, IPA-204, IPA-205 e IPA-206, procedentes dos municípios de Serra Talhada e Caruaru, bem como verificar a influência desses microrganismos na germinação das sementes. Foram analisadas 400 sementes de cada cultivar, pelo método do papel de filtro, que demonstrou a presença de 71 espécies fúngicas compreendidas em 23 gêneros. Os mais freqüentes foram Aspergillus, Penicillium e Fusarium, correspondendo a $81,44 \%$ do total de colônias encontradas. Com relação à qualidade das sementes, as maiores incidências foram observadas nas sementes cultivadas em Serra Talhada, possivelmente pelo tempo de armazenamento. Os fungos afetaram a germinação, principalmente, nas cultivares IPA-201 e IPA-202 de Serra Talhada com percentuais de germinação correspondentes a 53,2 e $65,5 \%$, respectivamente. A presença dos fungos foi observada nas sementes causando necrose nos cotilédones, radícula e folhas primárias, originando plântulas anormais. As sementes da cultivar IPA-206 apresentaram melhor padrão de sanidade e germinação.

Palavras-chave adicionais: identificação fúngica, sanidade, patologia de semente.

\section{ABSTRACT \\ Detection of endophytic fungi in cowpea seeds from Serra Talhada and Caruaru, Pernambuco, Brazil}

The purpose of the present work was to investigate and identify endophytic fungi in seeds of cowpea (Vigna unguiculata) cultivars, IPA-201, IPA-202, IPA-204, IPA-205, and IPA-206 from Caruaru and Serra Talhada counties, and evaluate the influence of those microorganisms on germination. Four hundred seeds of each cultivar were analyzed by blotter test method, which revealed the presence of 71 fungus species involving 23 genera. The most prevalent genera were Aspergillus, Penicillium and Fusarium, corresponding to $81.44 \%$ of all the colonies found. The highest contamination index was observed in the seeds from Serra Talhada, which shows an association between higher numbers of fungal colonies with longer storage time. The influence of microorganisms on germination was observed mainly in IPA-201 and IPA-202 cultivars from Serra Talhada, with a percentage corresponding to $53.25 \%$ and $65.50 \%$, respectively. The presence of fungi was observed in the seeds, causing necrosis in cotyledon, root and primitive leaf and generating abnormal seedlings. In the IPA-206 cultivar higher performance was shown in both seed health and viability.
O caupi [Vigna unguiculata (L.) Walp.] apresenta grande importância sócio-econômica para o Estado de Pernambuco. É uma cultura adotada basicamente por pequenos produtores rurais que utilizam a mão-de-obra familiar e que contribui para a permanência do homem no setor rural. Entretanto, esta cultura apresenta vários entraves, dentre os quais se destaca a falta de adoção de tecnologia, principalmente em relação aos insumos, onde o agricultor recorrendo ao uso de grãos como sementes, sem tratamento adequado, contribuindo assim para o surgimento de doenças.

A maioria dos agentes etiológicos das doenças do caupi é transmitida por sementes, principalmente as causadas por fungos que reduzem o poder germinativo e podem ser disseminados, estabelecendo assim focos primários de infecção em novas áreas de cultivo (Machado, 1994).

A semente é considerada um dos meios mais eficientes na disseminação de patógenos, os quais podem estar infestando-as ou infetando-as, dependendo da sua localização (Agarwal \& Sinclair, 1987). Após a infecção, geralmente os fungos xerófilos ou tolerantes às condições secas produzem propágulos de resistência, como clamidosporos, esclerócios ou micélios dormentes, capazes de permanecer viáveis por muito tempo nas sementes.

A má qualidade sanitária das sementes contribui para a sua baixa germinação, redução do número de plantas no estande, podridões radiculares, murchas e, conseqüentemente, 
Detecção de fungos endofíticos em sementes de caupi provenientes de...

baixo rendimento da cultura.

É grande a diversidade fúngica encontrada em sementes de caupi, principalmente gêneros como Penicillium Link., Aspergillus Link. e Curvularia Boedjn. Barros \& Menezes (1981) obtiveram uma frequência de $65,6 \%$ dos dois primeiros gêneros em 32 espécies de fungos detectados em caupi. Além destes, outros gêneros são comuns em sementes dessa cultura, como: Alternaria Ness., Botryodiplodia Sacc., Chaetomium Kunze: Fr., Cladosporium Link., Epicoccum Lk., Fusarium Link., Macrophomina Petr., Phomopsis Sacc., Rhizopus Ehr., Torula Pers., Trichoderma Pers., e Trichothecium Link. (Oliveira \& Bolkan, 1981). Dos 15 gêneros encontrados em sementes de caupi, por estes dois pesquisadores, apenas Macrophomina, Botryodiplodia, Phomopsis e Fusarium foram patogênicos. Porém, Torres et al. (1997) relataram que apesar da constante presença de Aspergillus niger Thiegh., A. flavus Link, Trichoderma sp. e Penicillium digitatum (Pers.: Fr.) Sacc., com freqüências de 51, 12, 2 e 1\%, respectivamente, os lotes de sementes analisados não mostraram seu vigor afetado.

Objetivando avaliar a população fúngica endofítica das sementes, bem como analisar o poder germinativo, foram utilizadas cinco cultivares, IPA-201, IPA-202, IPA-204, IPA205 e IPA-206, adquiridas da Empresa Pernambucana de Pesquisa Agropecuária (IPA), oriundas dos municípios de Serra Talhada, cultivadas no ano agrícola de 1995, e de Caruaru, cultivadas em 1996.

Amostras de 4.000 sementes de caupi foram inicialmente desinfestadas por $5 \mathrm{~min}$ em imersão de solução de hipoclorito de sódio $(\mathrm{NaOCl})$, a 1,5\% de cloro ativo, seguida de duas lavagens com água esterilizada. As sementes foram plaqueadas em caixas gerbox, previamente desinfestadas por exposição à luz ultravioleta (UV) durante $20 \mathrm{~min}$, contendo papel de filtro esterilizado e umedecido com $5 \mathrm{ml}$ de água destilada e estéril. O delineamento utilizado foi inteiramente casualizado em arranjo fatorial $5 \times 2 \times 20$, (cinco tratamentos, duas localidades e 20 repetições), sendo a unidade experimental representada por uma caixa gerbox contendo 20 sementes. As sementes foram incubadas em condições de fotoperíodo de $12 \mathrm{~h} \mathrm{luz/} 12 \mathrm{~h}$ escuro, a temperatura de aproximadamente $28^{\circ} \mathrm{C}$, durante sete dias. Após esse período, o percentual de germinação foi quantificado, considerandose como sementes germinadas aquelas que apresentaram emissão de suas estruturas principais, como radícula e caulículo (Rocha, 1975). Paralelamente, foi efetuado o levantamento da população fúngica das sementes. As colônias que se desenvolveram sobre as sementes foram transferidas para meio batata-dextrose-ágar (BDA), para posterior identificação. A identificação das espécies fúngicas foi realizada em microculturas, conforme Sutton (1980), com posterior montagem das lamínulas em lâminas, coradas com Azul de Amann, para observação das estruturas ao microscópio óptico.

Para viabilizar a identificação de algumas espécies de fungos como Penicillium e Aspergillus foi utilizado o meio específico Czapek Yeast Extract Ágar-CYA $\left(\mathrm{K}_{2} \mathrm{HPO}_{4}, 1,0 \mathrm{~g}\right.$;
Czapek concentrate, $10 \mathrm{ml}$; Yeast extract, 5,0 g; Sucrose, 30 g; Ágar, $15 \mathrm{~g}$; água destilada, $1000 \mathrm{ml}$ ). Esse substrato permitiu avaliar a coloração, reverso, borda, textura e diâmetro das colônias, e associar estas características à morfologia dos respectivos fungos (Pitt, 1985; Singh et al., 1991). Os demais isolados fúngicos foram identificados de acordo com as características morfológicas, seguindo-se a literatura específica disponível.

Os resultados obtidos em relação à população fúngica endofítica encontrada nas cultivares de caupi IPA-201, IPA202, IPA-204, IPA-205 e IPA-206 encontram-se na Tabela 1. Foram detectadas e identificadas 71 espécies de fungos compreendidas em 23 gêneros. Os que ocorreram com maior freqüência foram Aspergillus, Penicillium e Fusarium, os quais corresponderam a $81,44 \%$ do total de colônias obtidas. Dentre estes, os gêneros Aspergillus e Penicillium prevaleceram em $68,08 \%$ das colônias. Resultados semelhantes foram encontrados por Barros \& Menezes (1981), Oliveira (1981) e Torres et al.(1997). A alta freqüência desses organismos parece estar relacionada com a idade e com as condições de armazenamento das sementes, visto se tratarem de fungos de armazenamento. De acordo com as condições de armazenamento, esses fungos podem comprometer a qualidade das sementes, reduzindo assim o seu poder germinativo, ocasionando a morte de embriões (Oliveira, 1981).

Dentre as espécies de Aspergillus, A. niger foi a mais comum, apresentando frequência percentual de $14 \%$, seguida de $A$. ochraceus Wilhelm (8,68\%), A. parasiticus Speare (5,07\%), A. flavus $(4,56 \%)$, além de outras observadas com menor freqüência. As 14 espécies de Aspergillus identificadas têm sido comumente citadas como patógenos associados à semente de caupi, visto se tratarem de patógenos que suportam ambientes com baixa umidade. $O$ fungo permanece por muito tempo nas sementes e normalmente não causa doença em condições de campo, desempenhando entretanto, importante papel na deterioração das sementes durante o armazenamento, além de produzirem toxinas que afetam sua qualidade (Agarwal \& Sinclair, 1987).

Várias espécies de Penicillium foram identificadas, tendo a maioria das sementes apresentado alto grau de infecção. Apesar da constante presença destas espécies em levantamentos de microrganismos associados às sementes, a identificação ao nível de espécie tem sido pouco explorada. Neste sentido, no presente trabalho, registrou-se a presença de 15 espécies, algumas com alta frequência, como $P$. variable Sopp $(7,03 \%)$, $P$. funiculosum Thom $(4,18 \%)$ e $P$. oxalicum Currie \& Thom., (3,04\%). Algumas são citadas na literatura como fungos de armazenamento, especificamente $P$. oxalicum, P. purpurogenum Stoll, P. citrinum Thom, P. coryophilum Dierckx, P. crateriforme Gilman e Abbott, P. funiculosum, $P$. pinophilum Hedgcock, $P$. variabl, e P. felutano Biourge (Pitt, 1985; Singh et al., 1991), e outras como agentes causadores de doenças em vários hospedeiros. Penicillium olsonii Bain \& Sartory, P. implicatum Biourge e $P$. canescens Sopp são espécies que, apesar de serem relatadas como fungos de rara ocorrência na natureza, são detectadas principalmente em 
solo, madeira em decomposição e água, sendo dificilmente encontradas em sementes (Pitt, 1985).

Espécies do gênero Fusarium foram freqüentemente isoladas em todas as cultivares nas duas localidades. Entretanto, apenas sete espécies foram observadas: F. semitectum Berk. \& Rav. in Berkeley (6,33\%), F. equiseti (Corda) Sacc (2,98\%) F. oxysporum Schlecht.(2,22\%), F. solani (Mart.) Sacc. $(0,70 \%), F$. anthophilum (A. Braun) Wollen (0,32\%), $F$. sporotrichioides Sherbakoff $(0,19 \%)$, e F. moniliforme Sheldon (0,13\%). Em geral, as espécies de Fusarium relatadas neste trabalho causaram inibição na germinação de algumas sementes, e mesmo as que germinaram apresentaram crescimento do fungo sobre cotilédones e folhas primárias, além de necrose na radícula. Outras espécies fúnficas foram detectadas como Rhizopus stolonifer (Ehrenb.: Fr), Phomopsis sp., Trichoderma viride Pers. ex S. F. Gray Aggr. (Tabela 1).

Os dematiáceos ocorreram em baixa freqüência em relação aos demais fungos, correspondendo a 5,8\%, porém, com maior diversidade de espécies, dentre as quais destacaram-se Curvularia lunata (Wakker) Boedijn, Cladosporium elatum Harz., Alternaria alternata (Fr.:Fr.) Keissl., Alternaria sp., Nigrospora sphaerica (Sacc.) Mason, Drechslera ellisii Alcorn e Pithomyces sp. Berkeley. É possível que a alta incidência deste grupo de fungos seja devido a sua característica de formação de esporos com coloração escura e paredes espessas, as quais em geral, são mais resistentes às condições adversas, quando comparados com aqueles que apresentam esporos hialinos (Neergaard, 1977), podendo permanecer por muito tempo nas semente.

Dentre os fungos fitopatogênicos à cultura do caupi,

TABELA 1 - População fúngica endofítica associada à semente de cinco cultivares de caupi (Vigna unguiculata) dos municípios de Serra Talhada e Caruaru, Estado de Pernambuco

\begin{tabular}{|c|c|c|c|c|c|}
\hline Espécie & Colônia $\left(\mathbf{N}^{\mathbf{0}}\right)$ & Freqüência ${ }^{1}(\%)$ & Espécie & Colônia $\left(\mathrm{N}^{0}\right)$ & Freqüência $^{1}(\%)$ \\
\hline Aspergillus niger & 221 & 14,00 & F. anthophilum & 5 & 0,32 \\
\hline A. ochraceus & 137 & 8,68 & F.sporotrichioides & 3 & 0,19 \\
\hline A. parasiticus & 80 & 5,07 & F. moniliforme & 2 & 0,13 \\
\hline A. flavus & 72 & 4,56 & Rhizopus stolonifer & 103 & 6,25 \\
\hline A. aculeatus & 43 & 2,72 & Phomopsis sp. & 58 & 3,67 \\
\hline A. ustus & 25 & 1,58 & Trichoderma viride & 25 & 1,58 \\
\hline A. vesicolor & 9 & 0,57 & T. longibrachiatum & 1 & 0,06 \\
\hline A. terreus & 7 & 0,44 & Curvularia lunata & 31 & 1,96 \\
\hline A. tamari & 6 & 0,38 & C. pallescens & 6 & 0,38 \\
\hline A. ostianus & 5 & 0,32 & Curvularia sp. & 3 & 0,19 \\
\hline A. sydowi & 6 & 0,38 & C. senegalensis & 1 & 0,06 \\
\hline A. fumigatus & 4 & 0,25 & Cladosporium elatum & 12 & 0,76 \\
\hline A. awamori & 4 & 0,25 & C. britanicum & 2 & 0,13 \\
\hline A. ludwigii & 1 & 0,06 & C. orchidis & 1 & 0,06 \\
\hline Aspergillus sp. & 1 & 0,06 & C. oxysporum & 1 & 0,06 \\
\hline Penicillium variable & 111 & 7,03 & Cladosporium sp. & 1 & 0,06 \\
\hline P. funiculosum & 66 & 4,18 & Nigrospora sphaerica & 8 & 0,51 \\
\hline P. oxalicum & 48 & 3,04 & Drechslera ellisii & 7 & 0,44 \\
\hline P. pinophilum & 38 & 2,41 & D. pappendorfii & 4 & 0,25 \\
\hline P. felutano & 34 & 2,15 & Drechslera sp. & 1 & 0,06 \\
\hline P. citrinum & 30 & 1,90 & Pithomyces sp. & 5 & 0,32 \\
\hline P. rugulosum & 25 & 1,58 & Alternaria alternata & 4 & 0,25 \\
\hline P. olsoni & 15 & 0,95 & Alternaria sp. & 3 & 0,19 \\
\hline P. canescens & 14 & 0,89 & Helminthosporium australiensis & 1 & 0,06 \\
\hline P. coryophilum & 12 & 0,76 & Zygosporium gibbum & 1 & 0,06 \\
\hline P. implicatum & 10 & 0,63 & Monilia sp. & 9 & 0,57 \\
\hline P. digitatum & 6 & 0,38 & Rhizoctonia solani & 8 & 0,51 \\
\hline P. purpurogenum & 5 & 0,32 & R. bataticola & 2 & 0,13 \\
\hline P. thomi & 5 & 0,32 & Chaetomium globosum & 4 & 0,25 \\
\hline P. crateriforme & 4 & 0,25 & Pestalotiopsis guepini & 3 & 0,19 \\
\hline Penicillium sp. & 2 & 0.13 & Colletotrichum gloeosporioides & 3 & 0,19 \\
\hline Fusarium semitectum & 100 & 6,33 & Didymostilbe sp. & 1 & 0,06 \\
\hline F. equiseti & 47 & 2,98 & Eurotium sp. & 1 & 0,06 \\
\hline F. oxysporum & 35 & 2,22 & Phyllosticta sp. & 1 & 0,06 \\
\hline F. solani & 11 & 0,70 & Diplodia sp. & 1 & 0,06 \\
\hline Fu sarium $\mathrm{sp}$. & 11 & 0,70 & Não identificados & 7 & 0,44 \\
\hline Total de colônias & 1579 & & & & \\
\hline
\end{tabular}

${ }^{1}$ Frequência das espécies fúngicas expressa em percentagem, considerando-se a soma total das colônias obtidas 
Detecção de fungos endofíticos em sementes de caupi provenientes de...

destacou-se Rhizoctonia bataticola (Tassi) Goid., que corresponde à forma esclerocial de Macrophomina phaseolina (Tassi) Goid., agente causal da Podridão Cinzenta do Caule (Pio-Ribeiro \& Assis-Filho, 1997). Cerca de $0,44 \%$ dos isolados fúngicos não puderam ser identificados, devido à falta de esporulação.

Os resultados referentes à influência das espécies de fungos na germinação das sementes das cultivares de caupi encontram-se na Tabela 2. Considerando as cultivares, independente da região, observa-se que a IPA-206 apresentou maiores valores absolutos de germinação. Entretanto, dentro de cada localidade, as cultivares IPA-201 e IPA-202 apresentaram menor percentagem de germinação em relação às outras. Fazendo uma comparação entre a germinação das sementes de Serra Talhada (Sertão) e Caruaru (Agreste), evidencia-se melhor germinação nas cultivares procedentes dessa última região. Possivelmente, este fato esteja relacionado ao ano de produção das sementes (Serra Talhada, 1995; Caruaru, 1996), condições climáticas e tempo de armazenagem.

De um modo geral, apesar da alta percentagem de germinação, foi observado a presença de necrose na radícula e cotilédones das plântulas, demonstrando a influência negativa dos fungos no vigor das mesmas, principalmente, $A$. niger e $F$. oxysporum. As sementes que não germinaram, geralmente apresentavam-se totalmente recobertas por colônias de A. flavus, Phomopsis sp. e Fusarium sp. (Figura 1D, E, F). Observando-se ainda a localização dos fungos internamente, tanto nos cotilédones como no embrião (Figura
TABELA 2 - Efeito de fungos endofíticos na percentagem de germinação de sementes de cultivares de caupi (Vigna unguiculata) procedentes de Serra Talhada e Caruaru, Estado de Pernambuco

\begin{tabular}{cccc}
\hline \hline Cultivar & $\begin{array}{c}\text { Serra } \\
\text { Talhada (\%) }\end{array}$ & Caruaru (\%) & Média $^{1}$ (\%) \\
\hline IPA-206 & $94,25 \mathrm{a}^{1}$ & $91,00 \mathrm{a}^{1}$ & $92,62 \mathrm{a}$ \\
IPA-204 & $83,25 \mathrm{ab}$ & $72,25 \mathrm{~b}$ & $78,00 \mathrm{~b}$ \\
IPA-202 & $65,50 \mathrm{~cd}$ & $88,50 \mathrm{a}$ & $77,00 \mathrm{~b}$ \\
IPA-205 & $77,25 \mathrm{bc}$ & $68,00 \mathrm{~b}$ & $72,62 \mathrm{~b}$ \\
IPA-201 & $53,25 \mathrm{~d}$ & $88,25 \mathrm{a}$ & $70,75 \mathrm{~b}$ \\
\hline Média & $74,70 \mathrm{~B}$ & $81,70 \mathrm{~A}$ & \\
\hline DMS (5\%) & 15,29 & 15,29 & 10,81 \\
\hline
\end{tabular}

${ }^{1}$ Médias de quatro repetições para cada tratamento.

Médias seguidas da mesma letra minúscula na vertical e maiúscula, na horizontal, não diferem entre si, pelo teste de Tukey, a 5\%de probabilidade.

1A, B) e externamente na casca das sementes (Figura 1C).

A Figura 2 mostra o comportamento da população fúngica das sementes de caupi, sendo que Serra Talhada apresentou maior número de colônias em relação a Caruaru, ocorrendo diferença significativa entre as duas localidades (Figura 2A). Vale ressaltar que a precipitação pluviométrica em Serra Talhada foi maior $(140,83 \mathrm{~mm})$ que em Caruaru (70,87 mm), respectivamente em 1995 e 1996. Gonçalves \& Lima (1988) relacionaram sementes cultivadas em período chuvoso com maiores índices de infecção e menor freqüência

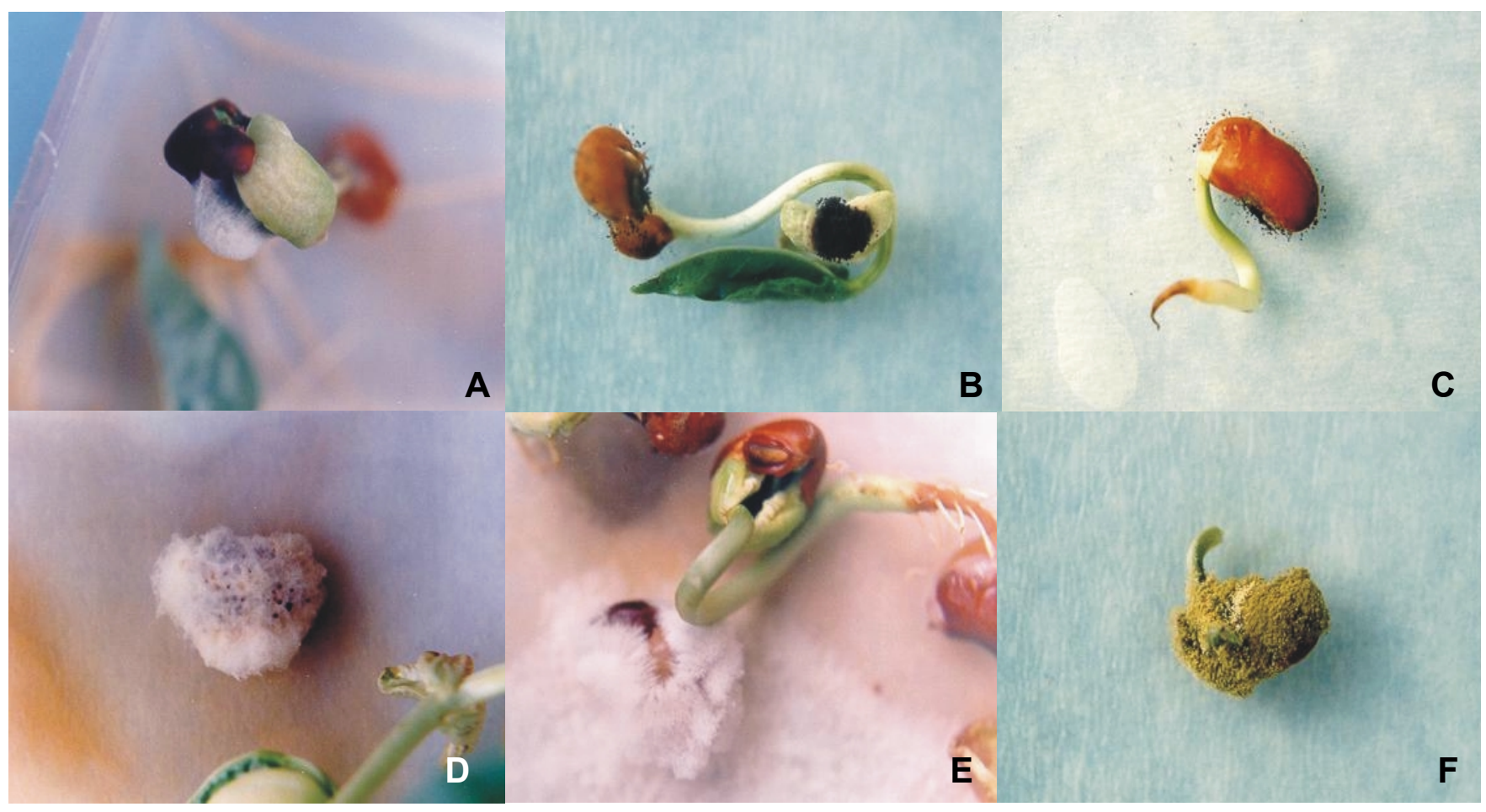

FIG. 1 - Localização de Fusarium oxysporum nas folhas primárias (A), Aspergillus niger nos cotilédones (B) e radícula (C) das plântulas, e efeito de Phomopsis sp. (D), Fusarium sp. (E) e Aspergillus flavus (F) na redução da germinação de sementes de caupi (Vigna unguiculata). 

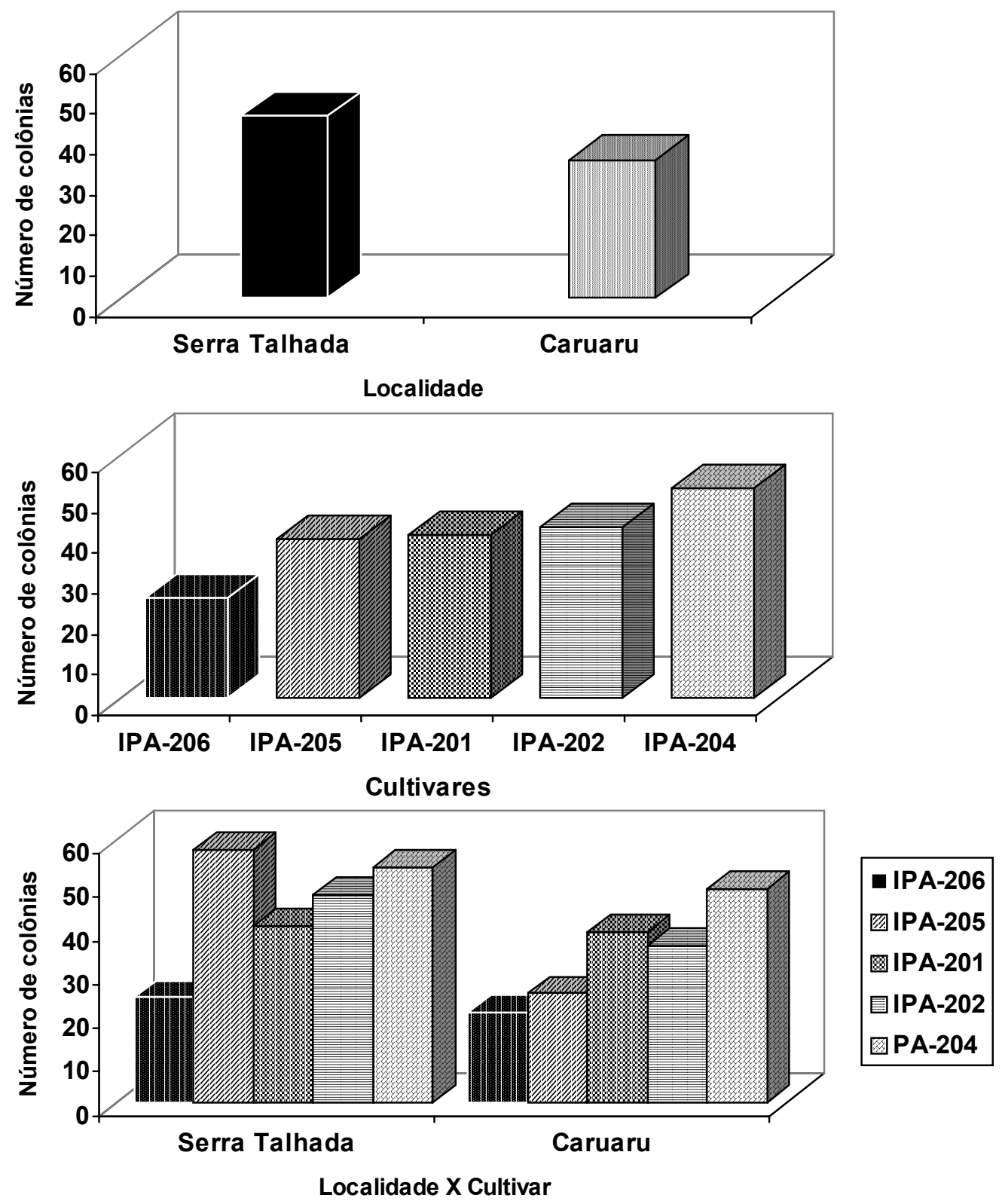

FIG. 2 - Análise da população fúngica obtida em sementes de cultivares de caupi (Vigna unguiculata), considerando a localidade (A), as cultivares (B) e a interação cultivar x localidade. Médias seguidas pela mesma letra não diferem estatisticamente entre si pelo teste de Tukey a 5\% de probabilidade.

de germinação, quando comparados com sementes produzidas em período seco.

A análise das populações distribuídas entre as cultivares mostrou que a cultivar IPA-206 apresentou menor número de colônias, não diferindo estatisticamente de IPA205 e IPA-201 (Figura 2B). Com relação a interação cultivar versus localidade, IPA-205 de Serra Talhada e IPA-204 de Caruaru apresentaram maior valor absoluto na percentagem de fungos (Figura 2C). É importante ressaltar a qualidade superior da cultivar IPA-206, tanto em Serra Talhada como em Caruaru, por apresentar maior percentagem de germinação e menor quantidade de fungos associados às sementes.

\section{REFERÊNCIAS BIBLIOGRÁFICAS}

AGARWAL,V.K. \& SINCLAIR, J.B. Principles of seed pathology. Boca Raton. CRC Press, 1987.

BARROS, S.T. de \& MENEZES, M. Fungos associados às sementes de feijão macassar, Vigna unguiculata (L.) Walp. procedentes do município de Caruaru, Estado de Pernambuco. Fitopatologia Brasileira 6:269-275. 1981.

FERREIRA, R.G. População fúngica em sementes de feijão, Phaseolus vulgaris L., fontes de resistência e controle químico a Fusarium oxysporum f. sp. phaseoli Kendrick \& Snyder (Tese de mestrado). Recife. Universidade Federal Rural de Pernambuco. 1983. 
Detecção de fungos endofíticos em sementes de caupi provenientes de...

GONÇALVES, N. de F.B. \& LIMA, J.A.A. Fungos associados a sementes de caupi produzidos em épocas distintas no Estado do Ceará. Ciência Agronômica 19:141-145. 1988.

MACHADO, J.C. Padrões de tolerância de patógenos associados à sementes. Revisão Anual de Patologia de Plantas 2:229-262. 1994.

MAUDE, R.B. Seedborne diseases and their control: principles and practice. Cambridge. CAB Internacional. 1996.

NEERGAARD, P. Seed pathology. London. UK. Macmillan Press. 1977.

NELSON, P.E., TOUSSON, T.A. \& MARASAS, W.F.O. Fusarium species: an illustrated manual for identification. University Park London. Pennsylvania State University Press. 1983.

OLIVEIRA, M.Z.A. Fungos associados a sementes de caupi: identificação, patogenicidade e controle. (Tese de Mestrado). Brasília. Universidade de Brasília. 1981.

OLIVEIRA, M.Z.A. de \& BOLKAN, H.A. Micoflora fúngica de sementes de dezoito variedades de caupi cultivadas em vários estados do Brasil. Fitopatologia Brasileira 6:576. 1981. (Resumo).
PITT, J.I. A laboratory guide to common Penicillium species. Sydney. CSIRO Division of Food Processing. 1985.

PIO-RIBEIRO, G. \& ASSIS-FILHO, F.M. Doenças do caupi. In: Kimati, H., Amorim, L., Bergamin Filho, A., Camargo, L.E.A., Resende, J.A.M. (Eds.) Manual de Fitopatologia. São Paulo: Agronômica Ceres. 1997. pp. 233-244.

ROCHA, F.F. Interpretação de testes de germinação. Brasília. Ministério da Agricultura-AGIPLAN. 1975.

SINGH, K., FRISVAD, J.C., THRANE, U. \& MATHUR, S.B. An illustrated manual on identification of some seed-borne Aspergilli, Fusaria, Penicillia and their mycotoxins. Denmark. Danish Government Institut of Seed Pathology for Developing Countries. 1991.

SUTTON, B.C. The appressoria of Colletotrichum graminicola and C. falcatum. Cannadian Journal of Botany 46:873-876. 1980.

TORRES, S.B., PEIXOTO, A.R. \& CARVALHO, I.M.S. Qualidade Sanitária e fisiológica de sementes de feijão-macássar (Vigna unguiculata (L.) Walp.) da região do submédio São Francisco. In: Resumos, Congresso Brasileiro de Sementes, 10, Curitiba, PR. 1997. p. 129. 